\title{
Stable and repeatable grasping of flat objects on hard surfaces using passive and epicyclic mechanisms
}

\author{
Vincent Babin ${ }^{\mathrm{a}}$, David St-Onge ${ }^{\mathrm{b}}$, Clément Gosselin ${ }^{\mathrm{c}}$ \\ ${ }^{a}$ Department of Mechanical Engineering, Université Laval, Québec, Qc, Canada G1V OA6 Email: vincent.babin.1@ @laval.ca \\ ${ }^{b}$ Department of Mechanical Engineering, Université Laval, Québec, Qc, Canada G1V 0A6 Email: david.st-onge.2@ulaval.ca \\ ${ }^{c}$ Department of Mechanical Engineering, Université Laval, Québec, Qc, Canada G1V 0A6 Email: Clement.Gosselin@gmc.ulaval.ca
}

\begin{abstract}
The stable and repeatable grasping of objects lying on a flat hard surface is addressed in this paper. A physical model of an object lying on a flat surface and its interaction with the environment and with a gripper is proposed. The important parameters governing the interaction are obtained. From this model, a grasping procedure is established and a robotic gripper is modified in order to grant the ability to pick up large thin objects lying on smooth hard surfaces. The procedure is implemented to demonstrate its repeatability on a chosen set of objects. It is shown that by sensing the force applied on the object and by taking advantage of the nature of the contact provided by the passive joint of the modified finger, a wide range of previously not directly graspable objects are made graspable via the application of a general approach. The experimental results reported clearly show the benefits of the simple force sensing implemented in the gripper as well as of the use of passive joints when interacting with very stiff environments. The proposed approach, while simple, yields a repeatable solution to a complex manipulation problem.
\end{abstract}

Keywords: Gripper, Robot Grasping, Constrained Grasping, Repeated Grasping, Passive Finger, Epicyclic Mechanism

\section{Introduction}

Applications of industrial robots are quickly expanding and so is their task definition. In most instances, the key component is their end-effector, either a specialised tool, a gripper or 5 a hand. Industrial grippers are often designed for a single task and are frequently made with tools that need to be replaced if the operation changes [1]. On the other hand, human inspired grippers, or robotic hands, are meant to cover a wider spectrum of tasks, but come at a higher price and are more complex to to operate. Simpler and more specialised grippers are present in industries such as food packaging, foundry manipulation, car assembly and are starting to appear in the agriculture industry to harvest vegetables or process them $[1,2]$.

The analytic investigation of grasping is being addressed by 15 many researchers for different finger designs in order to achieve a force closure on the object $[3,4,5,6]$. It was shown that a more stable grasp is achieved by a form closure instead [7, 8], i.e., a force closure without considering the friction forces. If human hands inspire dexterous robotic hands, the study of their taxonomy also helps the design of simple grippers [9], sometimes with the use of pattern recognition tools $[10,11]$. Indeed, $60 \%$ to $70 \%$ of human grasps use only two fingers [1]. It should also be noted that many analytically derived grasps - although theoretically possible - are not achievable due to the potential for ejection of the object [5] or interferences with the environment (occlusions).

This research targets a special case of object grasping that most simple grippers cannot achieve and that challenges dexterous robotic hands. Indeed, the grasping of a flat object on a flat surface is a complex task to perform without taking advantage of a nearby wall or edge. Specific solutions exist, such as electromagnetic grippers [12], which focus on generating artificial friction on a wider range of materials than metals but which are ill-suited for electrically sensitive objects such as electronic components. Other examples of tailored solutions are the use of vacuum grippers [13], which are less effective on uneven or porous surfaces, or the use of the universal gripper [14], which relies on positive and negative pressures to grasp parts with a membrane containing granular material. Soft fabrics in industrial plants can be manipulated with a suction gripper. However more versatile solutions are required in industry, i.e., to extend the abilities of simple two- or three-finger grippers for the grasping of flat objects. According to the most commonly used taxonomy, grasps are often categorised as power grasps, lateral grasps, precision grasps or adduction grasps. The power and adduction grasps are ill candidates for a task, such as picking up a flat object whose bottom face is hard to reach. However, the precision grasp, for instance using the tip of the fingers, is a possible solution, as shown in [15] and [16]. In the former case, the finger tips flip small objects, but this solution is quite restrictive regarding the object types and sizes. In the latter case, an underactuated two-finger gripper uses its compliance to transform the grasp in a parallel configuration, which is also greatly sensitive to the object size.

The type of grasp targeted in this study is the lateral grasp. In fact, the lateral grasp is one of the most common ways that humans use to grab objects, as observed in [17]. Following the study reported in [17], the authors introduced a hook finger designed for the lateral grasp showing great performances but 
60 restricting the thickness of the objects. An advantage of the lateral grasp is that it can be simplified to a planar grasp, since all forces are acting on a plane perpendicular to the flat surface, in which the fingers rest. It is worth mentioning that other strategies, relying on the environment more than on the finger design 65 itself, can also be used to address this problem. For instance, pushing the object toward the edge of a table to grasp it [18]. Another option is to generate a trajectory of the object to take advantage of its dynamics [19, 20, 21, 22]. Obviously, these types of strategies are less versatile because they either rely on 70 specific features other than the flat surface in the environment of require fast moving manipulators. Trivial solutions to the problem include the use of suction cups, which is a well known solution that cannot be used for some objects (e.g. porous surfaces) and the use of spatula-like devices to slide under the ob75 ject and lift it from the ground [23]. The fundamental issue with a spatula-like device is that it cannot be inserted under objects solely by moving towards them: it is necessary to have a reaction force that act on the object to maintain it in place. Section 2.2 explains mathematically why this happens. This reaction force can be the friction between the object and the surface. However, in most cases this strategy fails due to the surface being too smooth to generate the necessary friction as shown in Figure 1. Another solution would be to approach the object at

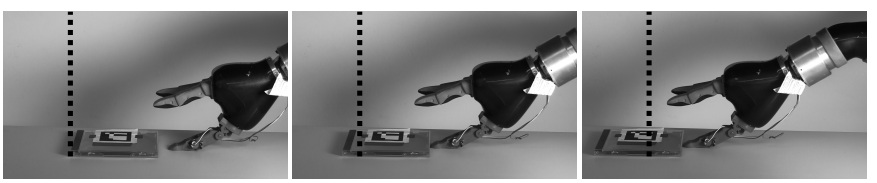

(a) Spatula-like thumb failing to insert underneath a rigid object (Rigid CD case)

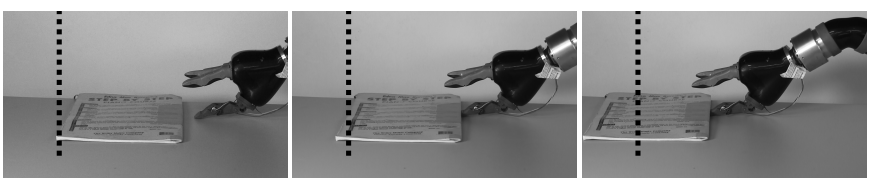

(b) Spatula-like thumb failing to insert underneath a flexible object (Flexible booklet)

Figure 1: Two examples a manipulator sliding a spatula-like thumb towards two objects (Rigid and Flexible) and failing to do so because of low friction between the object and the surface. The dotted line represents the initial position of each object.

great speed so that the inertia of the object maintains it in place some degree. This, however, could result in ejecting objects in the case of insertion failure. In [23] a finger-like mechanism is proposed that goes around the object effectively pushing the object onto the spatula-like platform. All of these solutions rely on the assumption that the objects fit entirely onto the spatula ${ }_{90}$ or at least the centre of mass of the object can be located onto the platform. The advantage of using a system that includes opposing fingers is the ability to hold the objects within the grip and orient them arbitrarily even though the centre of mass of the objects is outside the grip itself.

The goal of this study is to investigate the grasping of objects lying on levelled planes by means of rigid manipulation to obtain a final lateral grasp. A recent study [24] focuses on cases where the gripper exploits the environment constraints to bring the object in a state otherwise unattainable by gripper means alone. It then proceeds to validate these strategies by comparing them to measures of human grasping in controlled environments. This paper instead focuses on the model of the manipulation of a planar rectangular object of unknown size lying on a flat levelled surface and proposes a solution to reach a lateral grasp. The solution is then tested by fitting a custom finger design to the Jaco robot, shown in Figure 3. The original gripper is considered as a pinch gripper in order to emulate common industrial grippers and the enveloping capabilities of the Jaco hand (underactuation) are not considered. Figure 2 shows the grasping of a coffee cup with, (a) the original gripper and (b) the modified gripper to show that the basic grasping capabilities of the original gripper are preserved in the modified gripper. In-
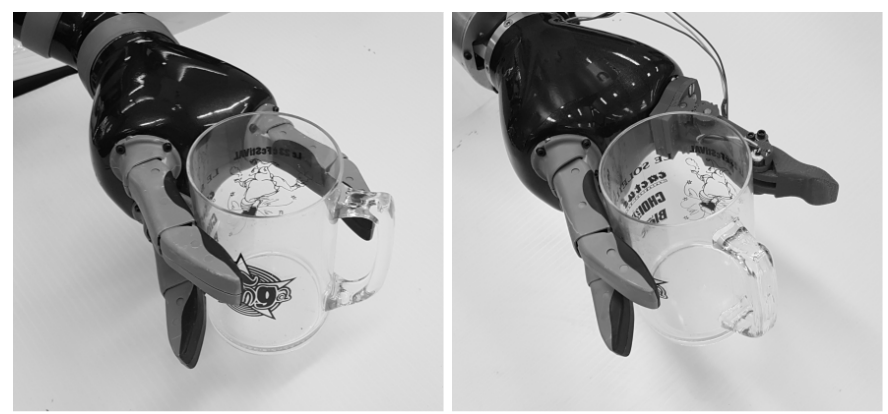

(a) Original gripper performing pinch (b) Modified gripper performing a grasp pinch grasp

Figure 2: The same grasping procedure performed by the original and modified gripper to demonstrate the equivalent basic capabilities of the new design compared to the original.

deed, it should be emphasised that the goal of this work is to get the fingers in opposition on each side of the object and not to study whether the final grasp is optimal or not. The proposed grasp procedure is then automated and performed by the proposed finger mechanism and the grasp process is shown to be repeatable. The contributions of this research are the analysis

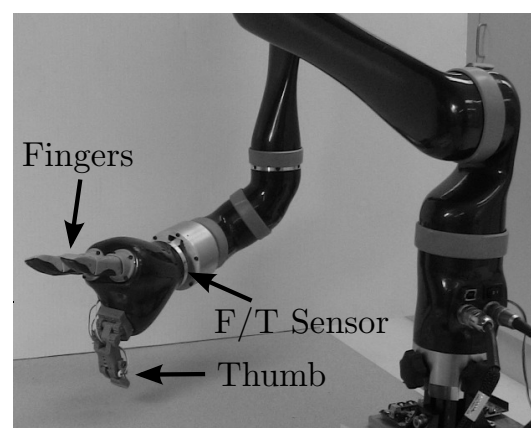

(a) Manipulator

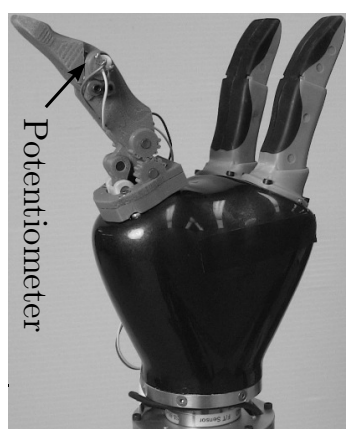

(b) Close-up of the gripper
Figure 3: The proposed mechanism mounted on a Jaco arm.

of the closed-form grasp for a flat object on a flat surface, the design of a novel finger and a closed-loop control experiment for a mechanical gripper. This paper is structured as follows. 
The interaction model of a finger with a flat object is first described in order to provide insight into the design choices of the novel finger, based on a thorough analysis. The relevant design parameters are then used to justify the design choices that led to the prototype. The results of the many grasping tests that were conducted with the prototype are then reviewed and the performances of the proposed finger and grasping strategy are assessed. Finally section 4 provides a discussion on the repeatability of the proposed solution.

\section{The proposed method}

As mentioned above, this work addresses the grasping of a flat object resting on a smooth flat surface. The main motivation for investigating constrained grasping problems is the need for robotic manipulators to grasp objects that are either too wide, too long or too thin for a direct top lateral grasp, considering the maximum opening $L_{\max }$ of a gripper as shown in Figure 4(a). Many common objects fall into this gripping category such as a sheet of paper, a book, a CD case or a credit card. Objects lying on flat surfaces are very common in real life or industrial environments and hence the relevance of this paper. The special solution of dynamically sliding a spatula-like finger under the object is discarded in this paper because it is considered that the manipulator must perform a task with the object. Therefore, simply supporting the object and lifting it up like in [23] does no suffice. Instead, this paper aims at developing a quasi-static method which is considered safer in cluttered environments. The proposed approach aims at bringing the gripper and object from an initial state to a final grasped state shown in Figure 4 and studies the conditions for which the transition from one state to the other is possible, i.e., to grasp an object of mass $(m)$, height $\left(h_{o}\right)$ and length $\left(l_{o}\right)$ as shown in Figure 4. An-

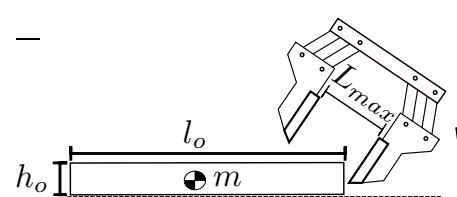

(a) Initial state

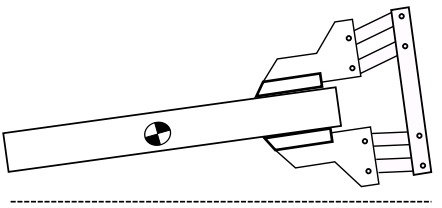

(b) Target state (Pinch Grasp)
Figure 4: Initial and target states.

other assumed property of the object in the static context of the grasp is that the object is rigid. Based on this assumption, the proposed interaction of the fingers with the object and the environment is shown Figure 5 where we consider a finger making contact with the top of the object while the thumb of the gripper is being forced between the object and the surface.

\subsection{Modelling of the grasping forces}

A trivial design of the proposed method would be to build a gripper which has a wide enough opening $L_{\max }$ to prevent the object from slipping away like in [23]. However since this is not always possible because of the need for compact grippers, the proposed solution prevents the objects from slipping away

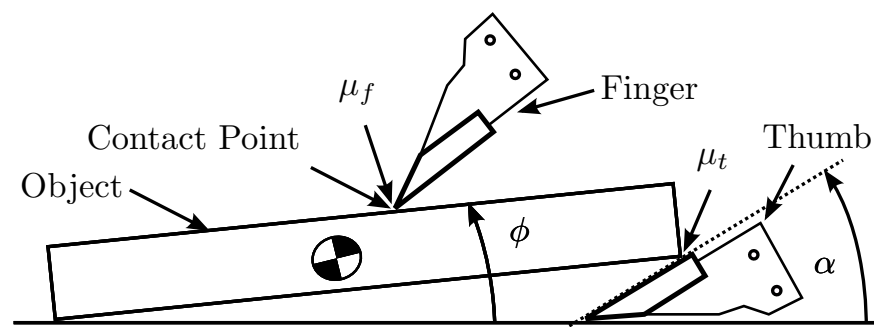

Figure 5: Table-wall Model.

Figure 5 shows the floating finger diagram of the object with the finger applying pressure at the contact point to hold the object in place while the thumb in being inserted under the object. Parameter $\phi$ is the object's angle relative to the resting surface, $\alpha$ is the thumb attack angle relative to the surface and $\mu_{t}$ is the friction coefficient between the thumb and the object. Figure 6

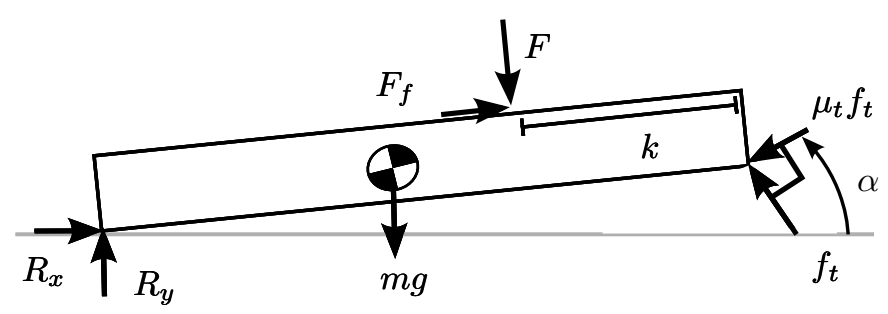

Figure 6: Free body diagram (FBD) of the object subjected to a finger force.

shows the Free body diagram (FBD) of the grasping approach with the friction force $F_{f}$ generated by a contact force $F$ exerted at a distance $k$ from the edge. Assuming a worst case scenario where there is no friction between the object and the surface, i.e. reaction force $R_{x}=0$, the only force able to counter the horizontal components of the forces $\mu_{f} f_{t}$ and $f_{t}$ are the horizontal components of forces $F$ and $F_{f}$. For this solution to work, the net force applied by the fingers (vector sum of $F$ and $F_{f}$ ) must remain within the friction cone, namely

$$
-\mu_{f} F \leq F_{f} \leq \mu_{f} F
$$

where $F$ is assumed to be greater than zero. The FBD of Figure 6 is used to obtain an expression for the friction force $F_{f}$ and determine the conditions on the parameters that increase the chances of Equation (1) to be valid, keeping in mind that friction coefficients are unknown hence the general approach. From the static equilibrium of the FBD of Figure 6, the friction force can be written as

$$
F_{f}=a F+b
$$

where

$$
\begin{aligned}
& a=\frac{s_{\phi}\left(c_{(\alpha-\phi)}-\mu_{t} s_{\alpha-\phi}\right)-\left(1-\frac{k}{l_{o}}\right)\left(\mu_{t} c_{\alpha}+s_{\alpha}\right)}{\frac{h_{o}}{l_{o}}\left(\mu_{t} c_{\alpha}+s_{\alpha}\right)-c_{\phi}\left(c_{(\alpha-\phi)}-\mu_{t} s_{(\alpha-\phi)}\right)} \\
& b=\frac{m g}{2} \frac{\left(c_{\phi}-\frac{h_{o}}{l_{o}} s_{\phi}\right)\left(\mu_{t} c_{\alpha}+s_{\alpha}\right)}{c_{\phi}\left(c_{(\alpha-\phi)}-\mu_{t} s_{(\alpha-\phi)}\right)-\frac{h_{o}}{l_{o}}\left(\mu_{t} c_{\alpha}+s_{\alpha}\right)} .
\end{aligned}
$$


with $c_{\phi}=\cos \phi, s_{\phi}=\sin \phi, c_{(\alpha-\phi)}=\cos (\alpha-\phi)$ and $s_{(\alpha-\phi)}=$ $\sin (\alpha-\phi)$. A graphical representation of the region defined by the inequalities of Equation (1) together with the line defined by Equation (2) is shown in Figure 7. On this graph, the static equilibrium (Equation (2)) defines a straight line of offset $b$ and slope $a$ while the friction constraint is the friction cone represented by the shaded area. The only variable remaining is the

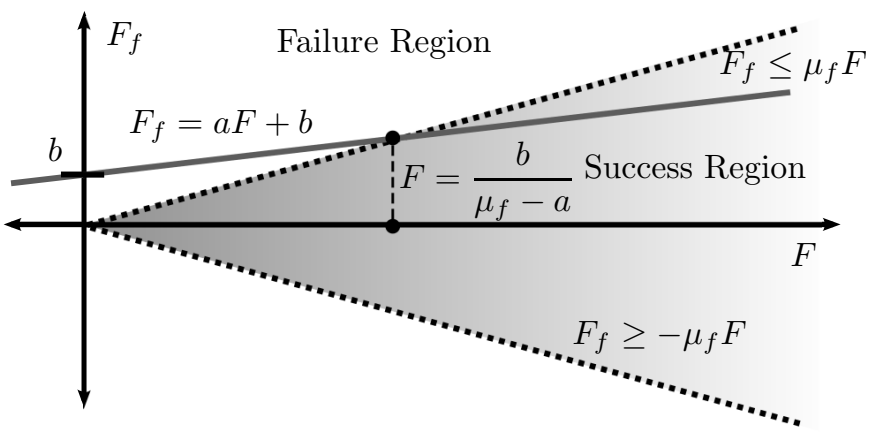

Figure 7: Feasibility regions and static constraint line in the $F, F_{f}$ plane.

force $F$, which determines whether the corresponding point on the line defined by Equation (2) is within the shaded area, i.e., whether the thumb can be successfully inserted.

\subsection{Analysis of the object-surface-thumb interactions}

By inspection of Figure 7, it can be observed that if the slope $a$ of Equation (2) satisfies $a \geq \mu_{f}$, it is impossible to enter the friction cone because the line defined by Equation (2) is either parallel to the friction cone or its slope is larger than that of the friction cone. This is easily seen by taking the upper bound of the inequality given in Equation (1) together with Equation (2) and solving for $F_{f}$ which yields

$$
F=\frac{b}{\left(\mu_{f}-a\right)} .
$$

Equation (5) gives the minimum finger pressure required to prevent object slippage effectively giving the minimum reaction as stated in the introduction to achieve static equilibrium. The case where no pressure is applied on the object, $F=0$, represents the case where the thumb is being inserted under the object but where the static equilibrium cannot be maintained, which means that the object slips away. It also means that decreasing $a$ increases the probability of a successful insertion. In Figure 7 and Equation (5) that the required force applied at the fingers is proportional to $b$ and hence $b$ must be as low as possible. This is an interesting observation because parameter $b$ is proportional to $m g$ which means that the proposed method is better suited for light objects which are in fact the objects that generate low friction between them and the surface and are therefore the most difficult to pick.

\subsubsection{Initial insertion}

In order to study the impact of the different variables on parameters $a$ and $b$, the feasible ranges for these variables are first set to the value of 0.5445 radians (31.2 degrees), which corresponds to the value of the thumb prototype presented later in this paper. Figure 8 shows that the maximum value that $\phi$ can reach is equal to $\alpha$, i.e., $\phi$ is limited to the range $0 \leq \phi \leq \alpha$. The material used for the prototype is ABS plastic and there-

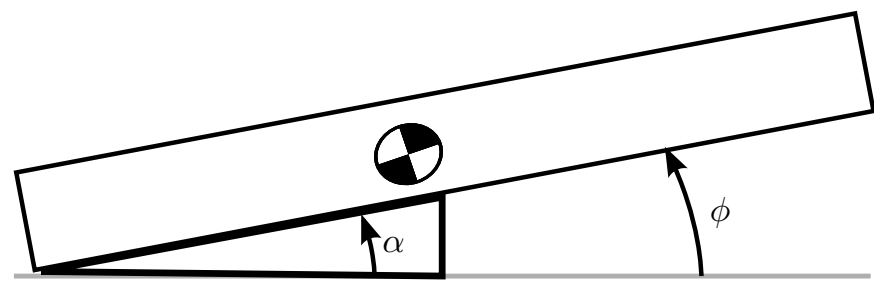

Figure 8: Extreme case for which $\phi=\alpha$.

fore the friction coefficient of ABS resin on carbon steel $\left(\mu_{t}=\right.$ 0.4 ) is used in the following numerical example [25]. Also, based on the tests performed with the prototype and presented in an upcoming section of this paper, the mass of the object being grasped is set to the maximum test sample value, which is $m=0.1667 \mathrm{~kg}$. Since $b$ is proportional to the mass (see Equation (4)), reducing the mass also reduces $b$, and hence the worst case to study is the heaviest object to be grasped. The remaining geometric parameters in Equations (3) and (4) are the finger contact distance, $k$, the height of the object, $h_{o}$ and the object length along the insertion plane, $l_{o}$. In order to study their impact, coefficients $a$ and $b$ are plotted as a function of angle $\phi$ in Figure 9 and Figure 10 respectively, for different values of the ratios $h_{o} / l_{o}$ and $k / l_{o}$.

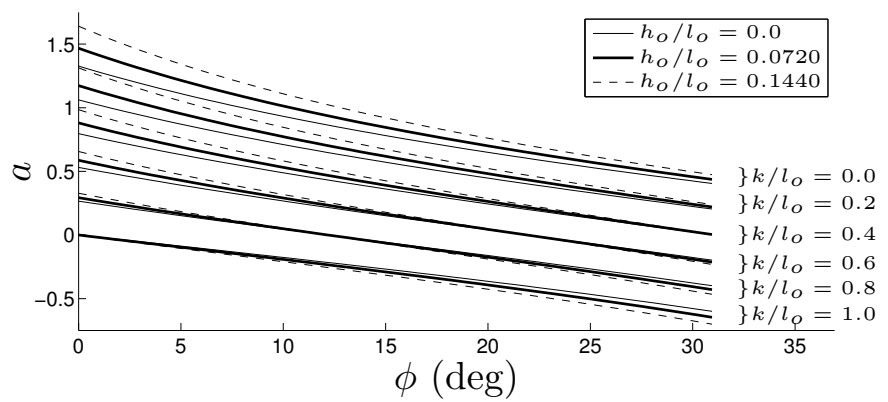

Figure 9: Coefficient $a$ as a function of angle $\phi$ for different values of the ratio $k / l_{o}$ and $h_{o} / l_{o}$

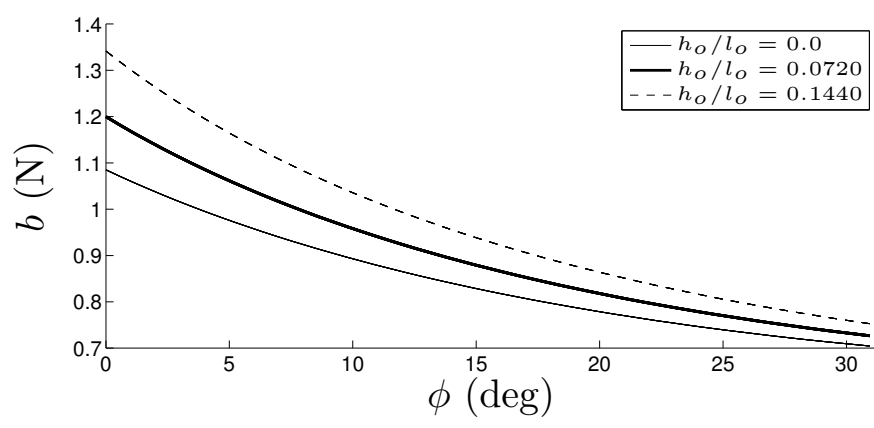

Figure 10: Coefficient $b$ as a function of angle $\phi$ for different values of the ratio $h_{o} / l_{o}$. Note that coefficient $b$ is independent from parameter $k$. 
It can be observed from Figures 9 and 10 that both parameters, $a$ and $b$, decrease as the thumb is being inserted (i.e. when $\phi$ increases), which means that as the thumb is being inserted, the required friction force between the fingers and the object decreases. This observation suggests that the focus should be placed on the influence of other parameters when $\phi=0$.

\subsubsection{Design parameters}

The impact of the design parameters on the capabilities of the gripper to perform a successful insertion is now investigated. The selected design parameters are $k, l_{o}$ and $\alpha$. Indeed, and $\alpha$ can be adjusted as part of the gripper design process while $l_{o}$ is affected by the choice of the grasping direction, i.e., the side of the object that is being grasped, which can be chosen as part of the grasp planning. To this end, parameters $a$ and $b$ are plotted as a function of angle $\alpha$ in Figures 11 and 12 for different combinations of $k / l_{o}$ and $h_{o} / l_{o}$.

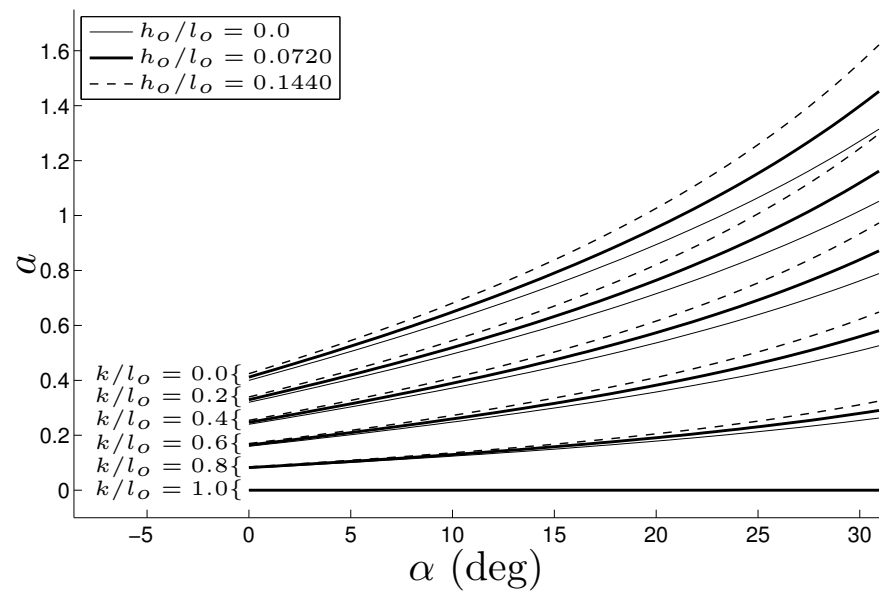

Figure 11: Parameter $a$ as a function of $\alpha$ with $\phi=0$, for different values of $k / l_{o}$ and $h_{o} / l_{o}$.

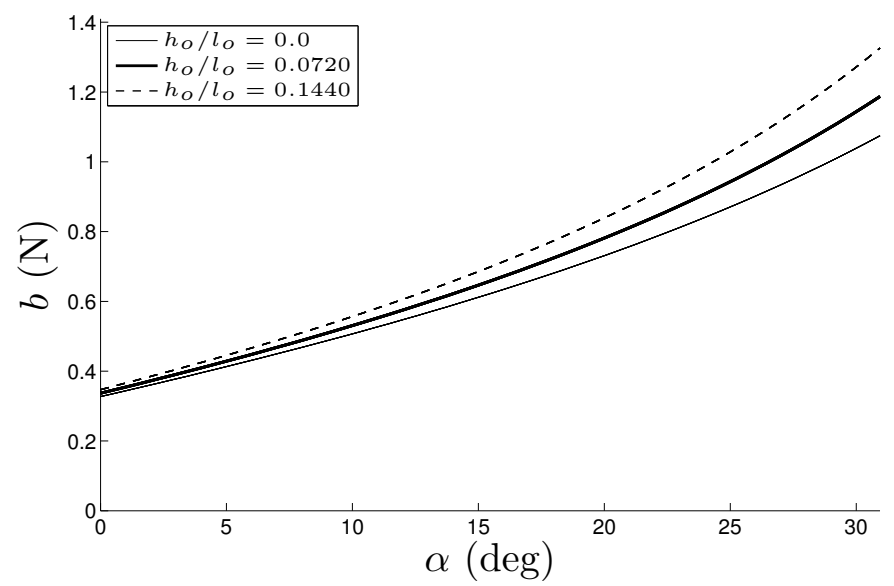

Figure 12: Parameter $b$ as a function of $\alpha$ with $\phi=0$, for different values of $h_{o} / l_{o}$.

The inspection of Figures 11 and 12 leads to the conclusion that reducing the ratio $h_{o} / l_{o}$, for instance by increasing $l_{o}$, reduces the value of $a$. This effect is negligible compared to the effect of reducing $l_{o}$ in the ratio $k / l_{o}$. Based on the assumption that reducing $a$ is decisive compared to reducing $b$, grasping the shortest side of the object $l_{o}$ is adopted. Following this, it is directly inferred that the design of the gripper must maximize $k$, minimize $\alpha$ and minimize $l_{o}$. Outlining that minimizing $\alpha$ is analogous to sharpening the thumb to approarch a spatula like object and also a trivial design decision, parameter $\alpha$ is again set constant to a value of 31.2 degrees in order to visualise the influence of $k / l_{o}$ and $h_{o} / l_{o}$ on parameters $a$ and $b$ in a continuous manner. The results are shown in Figures 13 and 14.

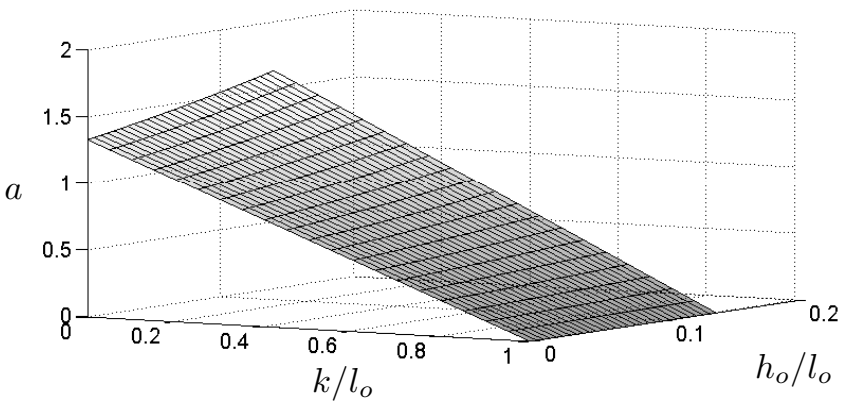

Figure 13: Parameter $a$ as a function of $k / l_{o}$ and $h_{o} / l_{o}$ for $\phi=0$ degrees.

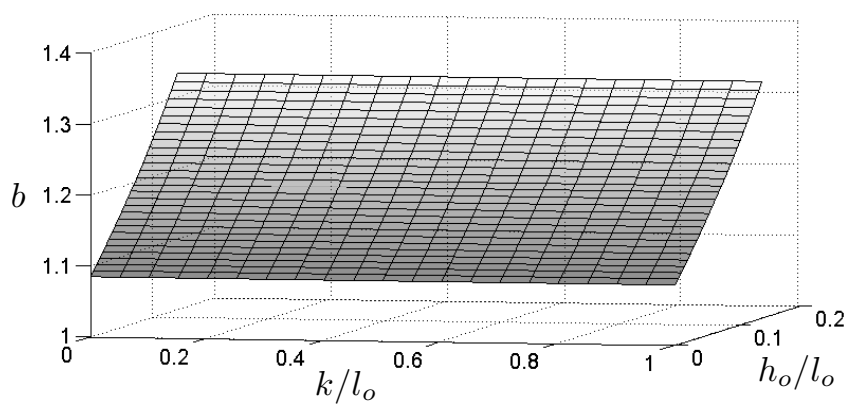

Figure 14: Parameter $b$ as a function of $k / l_{o}$ and $h_{o} / l_{o}$ for $\phi=0$ degrees

\subsection{The special case of flexible objects}

So far the model and the study have only considered objects of a very rigid nature. Everyday life objects range from the very flexible, like a sheet of paper, to the very rigid, in this study the Rigid CD case. The model used to investigate the case of flexible objects is the one shown in Figure 15. The model takes

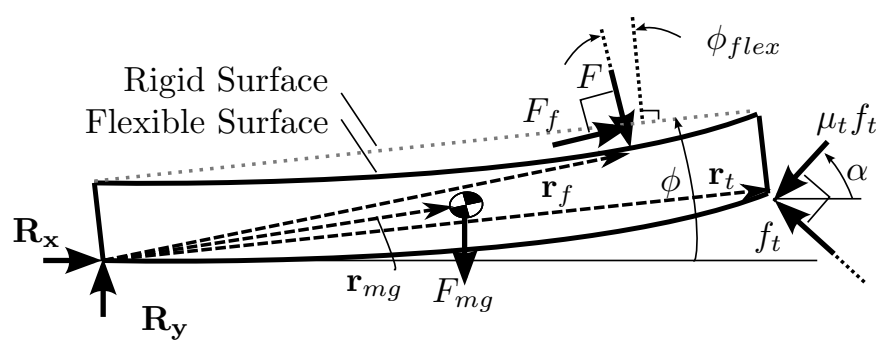

Figure 15: Free body diagram (FBD) of flexible object subjected to a pressure $F$. 
into account that an object under pressure will bend and hence the orientation of forces $F$ and $F_{f}$ will be rotated by angle $\phi_{\text {flex }}$ under the action of the force. Like in the previous section we aim at studying the friction force necessary to hold the object in place or rather how to minimise the necessary friction force. By assuming the reaction force $\mathbf{R}_{\mathbf{x}}$ equal to zero, we again study the case where the friction between the object and the surface is minimal. Taking the sum of forces in the $x$-axis, the sum of moments at the point of application of the reaction force $\mathbf{R}_{\mathbf{y}}$ and solving for the friction force $F_{f}$ we obtain a model similar to that of Equation 2 which is

$$
F_{f}=a_{f} F+b_{f}=\frac{c_{1} c_{5}-c_{3} c_{7}}{c_{2} c_{5}-c_{3} c_{6}} F+\frac{c_{3} c_{4}}{c_{2} c_{5}-c_{3} c_{6}}
$$

where $c_{1}=\left(\mathbf{Q}_{\phi} \mathbf{Q}_{\phi f l e x} \mathbf{e}_{y}\right)^{t} \mathbf{e}_{x}, c_{2}=\left(\mathbf{Q}_{\phi} \mathbf{Q}_{\phi f l e x} \mathbf{e}_{x}\right)^{t} \mathbf{e}_{x}, c_{3}=\left(\mathbf{Q}_{\alpha} \mathbf{e}_{\mu}\right)^{t} \mathbf{e}_{x}$, $c_{4}=\left(\mathbf{Q}_{\phi} \mathbf{r}_{m g} \times \mathbf{F}_{m g}\right)^{t} \mathbf{e}_{z}, c_{5}=\left(\mathbf{Q}_{\phi} \mathbf{r}_{t} \times \mathbf{Q}_{\alpha} \mathbf{e}_{\mu}\right)^{t} \mathbf{e}_{z}, c_{6}=\left(\mathbf{Q}_{\phi} \mathbf{r}_{f} \times\right.$ $\left.\mathbf{Q}_{\phi} \mathbf{Q}_{\phi f l e x} \mathbf{e}_{x}\right)^{t} \mathbf{e}_{z}, c_{7}=\left(\mathbf{Q}_{\phi} \mathbf{r}_{f} \times \mathbf{Q}_{\phi} \mathbf{Q}_{\phi \text { flex }} \mathbf{e}_{y}\right)^{t} \mathbf{e}_{z} . \mathbf{Q}_{\phi}, \mathbf{Q}_{\alpha}$ and $\mathbf{Q}_{\phi \text { flex }}$ are rotation matrices of angles $\phi, \alpha$ and $\phi_{\text {flex }}$ along the $z$-axis, $\mathbf{e}_{x}, \mathbf{e}_{y}, \mathbf{e}_{z}$ unitary vectors in the directions of the $x, y$ and $z$-axis,

$250 \quad \mathbf{F}_{m g}=\left[\begin{array}{lll}0 & -m g & 0\end{array}\right]^{t}$ and finally $\mathbf{e}_{\mu}=\left[\begin{array}{lll}-\mu_{t} & 1 & 0\end{array}\right]^{t}$. The flexible model obtained in Equation 6 is almost the same as the rigid model obtained in Equation 2, except expressed in vector form. When angle $\phi_{\text {flex }}$ is zero, Equation (2) and Equation (6) yield the same result hence the difference being only the rotation matrix $Q_{\phi \text { flex }}$ that appears in coefficients $c_{1}, c_{2}, c_{6}$ and $c_{7}$. Inspecting the coefficients, it can be seen that increasing the value of $\phi_{\text {flex }}$ is analogous to rotating forces $F$ and $F_{f}$ hence pushing the object with greater force toward the thumb than in the rigid case. According to this reasoning we conclude that studying the objects for rigid cases takes into account the flexible cases as $\phi_{\text {flex }}$ and hence the design guidelines of section 2.2.2 are also valid for flexible objects.

\section{Experimental Validation}

\subsection{Prototype design and modelling}

A gripper prototype was designed to validate the proposed grasping approach. Instead of developing a complete two- or three-finger gripper from scratch, the commercially available gripper of the 6-dof Jaco robotic arm was modified. The robot is also retrofitted with a force/torque sensor mounted at the wrist, as shown in Figure 3. Figure 3(a) shows the manipulator with the custom thumb installed and Figure 3(b) shows a close-up of the modified gripper.

The first issue to be addressed in order to apply the grasping strategy developed above is the precision required on the position of the inserted thumb. Indeed it was assumed above that the last phalanx of the thumb is resting on the surface on which the object is placed. If the thumb is too high, the object is pushed away while if the thumb is too low, large contact forces may be induced, which can damage the gripper. The solution proposed here is to introduce compliance with a spring-loaded passive distal phalanx. A schematic describing the principle of the proposed finger, including the distal phalanx, is shown in Figure 16. Using this mechanism requires that the manipulator initiate contact with the surface to push the distal phalanx

to the correct angle. The spring then ensures that the tip of the finger $P$ remains in contact with the surface. Moreover, in order to obtain some information on the environment with which the manipulator is in contact, the distal phalanx's angle relative to the proximal phalanx, noted $\theta_{3}$ in Figure 16(c), is measured by a potentiometer. This allows the manipulator to adjust to the surface height, to detect object edges and prevent damage to the mechanism by preventing the manipulator from trying to bend the phalanx out of its mechanical limits. In addition to the

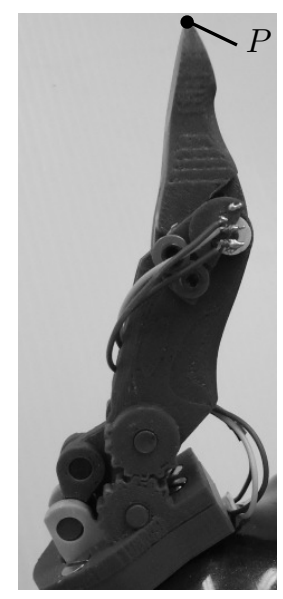

(a) Prototype thumb

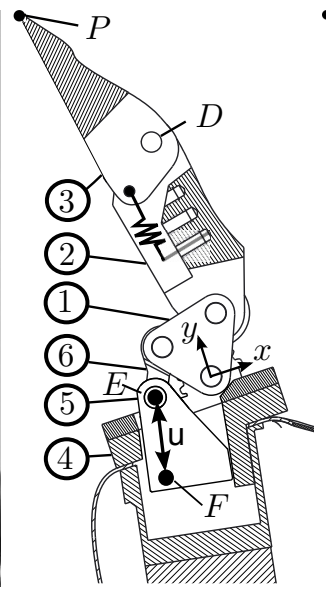

(b) Cut-view of the prototype thumb

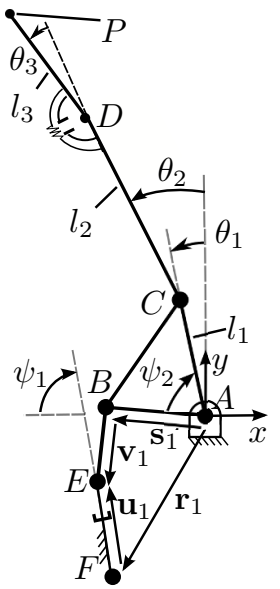

(c) Schematic of the prototype thumb

Figure 16: (a) JACO Hand with prototype thumb (b) Close-up of the modified thumb with (1) Planetary rack (2) Proximal phalanx (3) Distal phalanx (4) Hand frame (5) Linear actuator (6) Input link (c) Schematic with geometric parameters.

compliant distal phalanx, the second important feature of this thumb is the use of a rolling joint - or epicyclic mechanism - to increase the range of motion of angle $\theta_{1}$. Figures 16(c) and 17(b) show the different variables used to model the kinematics of this mechanism. The notation used in [26] is adopted

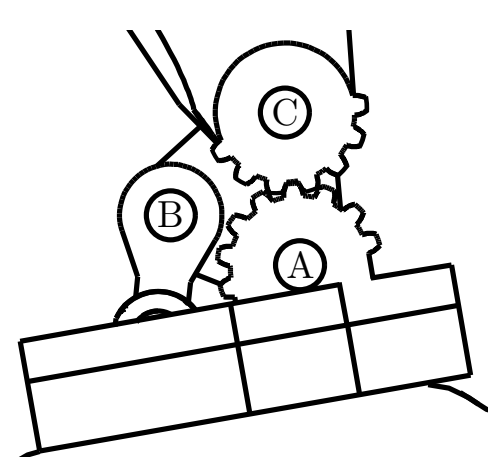

(a) CAD view of the planetary gear system (b) Schematic of the planetary gear system

Figure 17: Schematics of the prototype thumb

here to describe the mechanism. The mechanism is essentially a planetary geared system where the sun gear is fixed to the hand frame. The input is the carrier link and, in this case, the output is the planet gear which is also the proximal phalanx of 
the thumb. The gear ratio is therefore given by

$$
n=\frac{r_{1}}{r_{1}+2 r_{2}}
$$

where $r_{1}$ and $r_{2}$ are the radii of the pitch circles of respectively the sun gear and planet gear (or alternatively the number of teeth of each gear). Following this, the input-output angle relationship between angles $\theta_{1}$ and $\theta_{2}$ can be obtained as

$$
\theta_{2}=\frac{(n+1)}{(1-n)} \theta_{1}+\theta_{d}
$$

where $\theta_{1}$ and $\theta_{2}$ are respectively the carrier angle and proximal phalanx angle shown in Figure 16 and $\theta_{d}$ is a design parameter corresponding to the value of $\theta_{2}$ when $\theta_{1}$ is zero (initial offset). The input of the epicyclic mechanism, $\theta_{1}$, is in turn driven by a prismatic actuator through a slider-crank mechanism, as illustrated in Figure 16(c). The kinematics of this system can be solved using the loop constraint equation, namely loop $A B E F A$ of the slider-crank mechanism in Figure 16(c). The loop closure equation can be written as

$$
\mathbf{0}_{2}=\mathbf{r}_{1}+\mathbf{u}_{1}-\mathbf{v}_{1}-\mathbf{s}_{1}
$$

where $\mathbf{0}_{2}$ stands for the two-component zero vector, vector $\mathbf{r}_{1}$ is the position vector of the base of the actuator with respect to point $A, \mathbf{u}_{1}$ is the vector along the prismatic actuator, $\mathbf{v}_{1}$ is the vector connecting point $B$ of link $A B C$ to point $E$ (end point of the prismatic actuator) and $\mathbf{s}_{1}$ is the vector connecting point $A$ to point $B$, on link $A B C$. Solving Equation (9) for $\mathbf{v}_{1}$ and multiplying by its transpose knowing that $\mathbf{v}_{1}{ }^{T} \mathbf{v}_{1}=l_{6}{ }^{2}$, one obtains

$$
l_{6}^{2}=\left(\mathbf{r}_{1}+\mathbf{u}_{1}-\mathbf{s}_{1}\right)^{T}\left(\mathbf{r}_{1}+\mathbf{u}_{1}-\mathbf{s}_{1}\right)
$$

with $\mathbf{s}_{1}=l_{4}\left[-\sin \left(\theta_{1}+\psi_{2}\right) \cos \left(\theta_{1}+\psi_{2}\right)\right]^{T}, \mathbf{u}_{1}=\sigma \mathbf{e}_{1}, \mathbf{e}_{1}=$ $\left[\begin{array}{ll}-\cos \left(\psi_{1}\right) & \sin \left(\psi_{1}\right)\end{array}\right]^{T}$ and $\mathbf{r}_{1}=\left[\begin{array}{ll}r_{x} & r_{y}\end{array}\right]^{T}$ where $\psi_{1}$ and $\psi_{2}$ are the construction angles shown in Figure 16(c) and $\sigma$ is the extension of the prismatic actuator (input). Solving Equation (10) for the input $\sigma$ and selecting the proper branch of solution of the quadratic equations, one then obtains

$$
\sigma=\frac{1}{2}\left(\left(-2 \mathbf{e}_{1}^{T} \mathbf{c}\right)-\sqrt{\left(2 \mathbf{e}_{1}^{T} \mathbf{c}\right)^{2}-4\left(\mathbf{c}^{T} \mathbf{c}-l_{6}{ }^{2}\right)}\right)
$$

with $\mathbf{c}=\mathbf{r}_{1}-\mathbf{s}_{1}$. With this result, and with a prescribed value of $\theta_{2}$, angle $\theta_{1}$ is first obtained from Equation (8), then $\mathbf{s}_{1}$ is computed and finally Equation (11) is used to compute the required actuator position $\sigma$. Differentiating Equation (10) with respect to time gives

$$
\mathbf{e}_{1}^{T}\left(\mathbf{r}_{1}+\mathbf{u}_{1}-\mathbf{s}_{1}\right) \dot{\sigma}=\mathbf{d}_{1}^{T}\left(\mathbf{r}_{1}+\mathbf{u}_{1}-\mathbf{s}_{1}\right) \dot{\theta}_{1}
$$

with $\mathbf{d}_{1}^{T}=l_{4}\left[-\cos \left(\theta_{1}+\psi_{2}\right)-\sin \left(\theta_{1}+\psi_{2}\right]^{T}\right.$. Finally the input to output velocity relationship comes from differentiating Equation (8) to express $\dot{\theta}_{1}$ in terms of $\dot{\theta}_{2}$, yielding

$$
\frac{\dot{\theta}_{2}}{\dot{\sigma}}=\frac{\mathbf{e}_{1}^{T}\left(\mathbf{r}_{1}+\mathbf{u}_{1}-\mathbf{s}_{1}\right)}{\mathbf{d}_{1}^{T}\left(\mathbf{r}_{1}+\mathbf{u}_{1}-\mathbf{s}_{1}\right)} \frac{(n+1)}{(1-n)} .
$$

\subsection{Experimental procedure}

An experimental procedure was developed in order to validate the feasibility of the proposed grasping method in a real world setting. The experimental set-up is shown in Figure 18. Aruco markers [27, 28] are used as a means of testing the repeatability of the algorithm with consecutive tests. The vision system is used with two markers, namely: $i$ ) The Robot Marker which is fixed on the world reference frame at the base of the robot and ii) a second marker which is placed on the objects with its $z$-axis perpendicular to the surface of the objects and its $y$-axis pointing along the shorter side of the object. In the proposed scenario, only the length of the object along the $y$-axis is known. The markers are always placed as far as possible from the insertion edge, adjacent to the opposing edge. Knowing the marker sizes, the insertion edge's position can be determined and the contact distance $k$ of the fingers can be controlled.

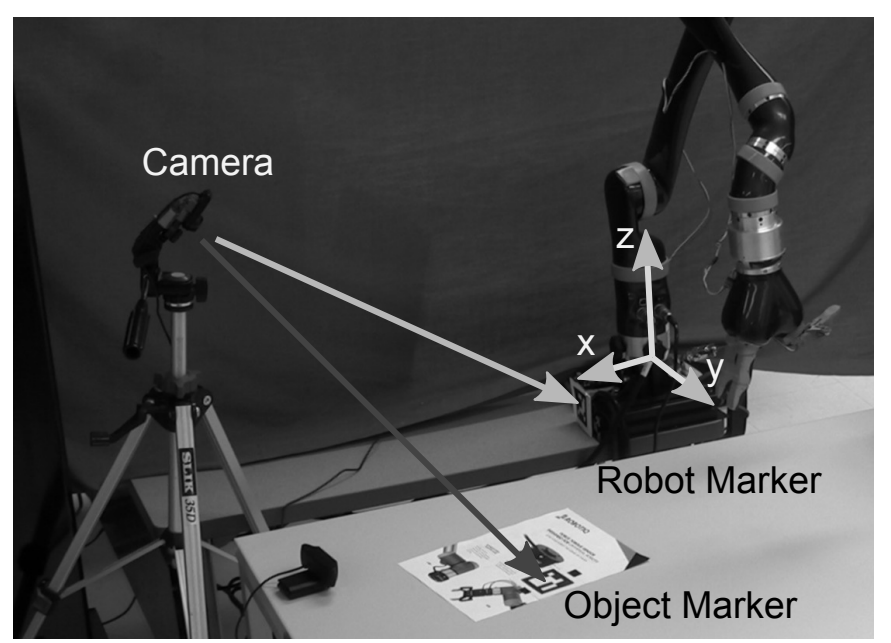

Figure 18: Test set-up with vision system and Aruco markers.

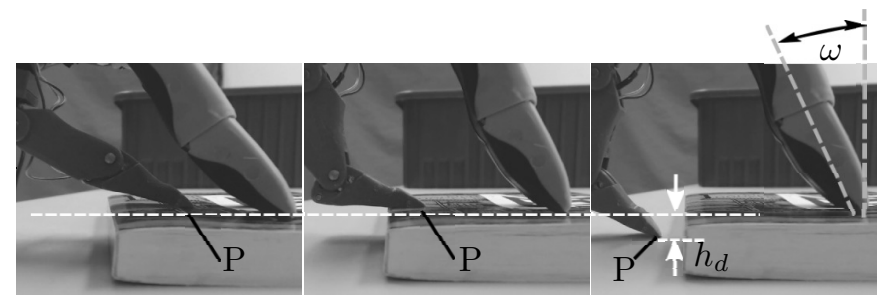

Figure 19: Additional parameters: attack angle $\omega$ and vertical offset $h_{d}$.

The grasping procedure implemented focuses on successfully inserting the thumb under the object and hence most of the explanations given in this section emphasise this part of the procedure. The first step is to bring the manipulator to a pre-set position in joint-space. The following step is for the gripper to position the tip of the finger over the contact point with an attack angle $\omega$ shown in Figure 19, which is the angle of the finger relative to the marker's $z$-axis along the principal axis of the object, that is set to a value $\omega_{i}$ and with the thumb's initial position input $\sigma$ set to a value $\sigma_{i}$. The fourth step is for the manipulator to lower the hand, keeping it at a constant orientation, stopping when a force along the $z$-axis of the world frame 
is measured to be of greater magnitude than a pre-set threshold $F_{0}$. The following step is to apply a force $F_{1}$ while decreasing $\sigma$ (finger actuation input) and adjusting $\omega$ to keep point $D$ (see Figure 16(c)) at a constant height along the $z$-axis of the world frame. This produces a motion of point $P$ towards the edge of the object. While this motion is taking place, the potentiometer at joint $\theta_{3}$ is monitored and the following condition is checked

$$
\left\|\theta_{3}(i)-\theta_{3}(i-1)\right\| \geq \theta_{f}
$$

where $\theta_{3}(i)$ stands for the value of $\theta_{3}$ given by the potentiometer at time $i$ and $\theta_{f}$ is a threshold that corresponds to an edge detection. An edge is detected when condition (14) is satisfied. If an edge is detected, a constant force $F_{1}$ is applied while the manipulator is adjusting $\omega$ to lower $D$ by an amount that corresponds to the drop of point $P$ that has been detected by the edge detection algorithm. Additionally, the following condition is also tested

$$
\theta_{3}(i) \leq \theta_{3, \max }
$$

where $\theta_{3, \max }$ is the maximum value allowed for the passive joint coordinate $\theta_{3}$. When Equation 15 is not satisfied, the limit value of the passive joint $\theta_{3}$ has been reached. This normally corresponds to a situation in which the object is too thin for the edge to be detected or a situation in which the edge was not reached due to a poor placement of the gripper relative to the object. The two possible different behaviours are compared in Figures 20 and 21. In the former case (Figure 20), the edge is detected, the height of the object is compensated for and the grasping action is performed. In the latter case (Figure 21), the limit of the passive joint is reached without detecting an edge because the object is too thin. The grasping action is performed when the maximum value of $\theta_{3}$ is reached.

The insertion step is similar to the thumb opening step, namely a constant force $F_{1}$ is being applied while the manipulator adjusts $\omega$ in order to keep point $D$ at a constant height. ever this step consists in inserting the thumb between the surfac and the object while requesting $\sigma$ to reach a set value $\sigma_{f}$. Once $\sigma=\sigma_{f}$, the final grasping sequence is performed. The final grasping sequence raises the gripper along the world frame $z$-axis while requesting the thumb and finger to reach a set ger are deactivated to prevent overheating of the motors. Since their mechanisms are self-locking, the pressure on the object is maintained. Any auxiliary task can then follow, for instance, in this experiment, releasing the object in a bin. The tasks are

1. Ready Position: Request the manipulator to go to a preset position in the joint space.

2. Marker Trigger: Wait for the vision system to provide the position and orientation of the object.

3. Pre-grasp position: Position the manipulator's finger at a distance $k$ from the edge and attack angle $\omega=\omega_{i}$ so that point $D$ is at an acceptable height when the finger makes contact with the object.

4. Lower: Request the manipulator to lower along the $z$-axis in the world frame.
5. Force Trigger: A force of magnitude $F_{0}$ is measured.

6. Open Thumb: Apply a downward force $F_{1}$ at the fingers, decrease $\sigma$ and keep point $D$ at a constant height.

7. Edge Detection: Check the inequality condition given in Equation (14).
8. Maximum Amplitude: Check the inequality condition given in Equation (15).

9. Adapt: Increase $\omega$ to lower point $D$ by a distance $h_{d}$ shown in Figure 19.

10. Close Thumb: Apply a downward force $F_{1}$ at the fingers while increasing $\sigma$ up to a value $\sigma_{f}$ and keeping point $D$ at a constant height.

11. Final grasping: Request finger and thumb to go to a predetermined position while keeping the hand at a constant orientation and raising it.

12. Auxiliary Task.

\subsection{Experimental parameters and results}

The procedure presented above was implemented and the numerical values of the different set-up parameters are now given. Table 1 shows the different values characterising the gripper while Table 2 provides the properties of the objects used in the tests. Using these parameters, the prototype thumb's

\begin{tabular}{|c|c|c|c|}
\hline \multicolumn{4}{|c|}{ Prototype Parameters } \\
\hline Parameter & Value (deg) & Parameter & Value $(\mathrm{mm})$ \\
\hline$\alpha$ & 31.20 & $\overline{l l_{1}}$ & 15.00 \\
\hline$\theta_{d}$ & 22.28 & $l_{2}$ & 43.20 \\
\hline$\psi_{1}$ & 100.00 & $l_{3}$ & 43.80 \\
\hline$\psi_{2}$ & 63.25 & $l_{4}$ & 13.50 \\
\hline$\omega_{i}$ & 28.00 & $l_{6}$ & 12.00 \\
\hline$\theta_{3, \max }$ & 110.67 & $r_{x}$ & -15.90 \\
\hline$\theta_{f}$ & 3.52 & $r_{y}$ & -10.60 \\
\hline Parameter & Value $(\mathrm{N})$ & $\sigma_{i}$ & 18.46 \\
\hline$F_{0}$ & 2.00 & $\overline{\sigma_{f}}$ & 20.37 \\
\hline$F_{1}$ & 3.00 & $r_{1}$ & 7.50 \\
\hline & & $r_{2}$ & 7.50 \\
\hline
\end{tabular}

Table 1: Experimental set-up properties

kinematics are shown in Figures 23 and 24.

Table 2: Object properties

\begin{tabular}{|l|r|r|r|r|}
\hline \multicolumn{5}{|c|}{ Object Properties } \\
\hline Description & $h_{o}(\mathrm{~mm})$ & $l_{o}(\mathrm{~mm})$ & $m(\mathrm{~kg})$ & $k(\mathrm{~mm})$ \\
\hline \hline Booklet & 3.05 & 229 & 0.1646 & 110 \\
\hline Pamphlet & 0.59 & 88 & 0.0124 & 80 \\
\hline Book & 14.00 & 128 & 0.1667 & 80 \\
\hline Flexible CD Case & 5.00 & 124 & 0.0368 & 110 \\
\hline Rigid CD Case & 10.38 & 124 & 0.0765 & 110 \\
\hline Sheet of Paper & 0.13 & 209 & 0.0108 & 80 \\
\hline
\end{tabular}




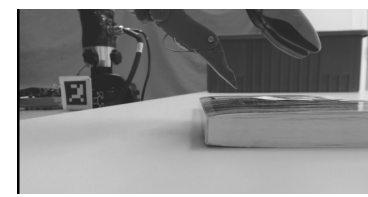

(a)

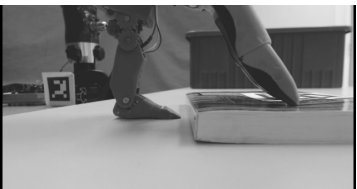

(f)

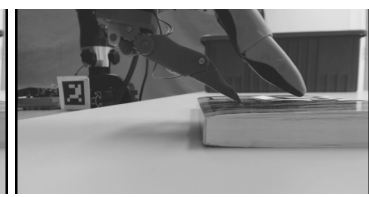

(b)

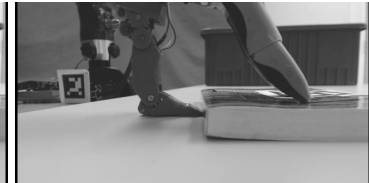

(g)

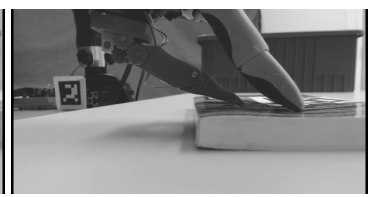

(c)

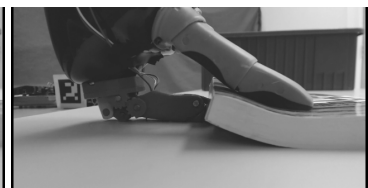

(h)

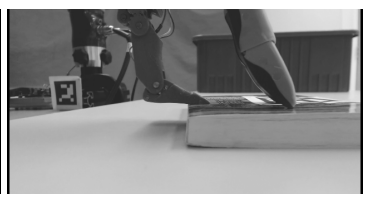

(d)

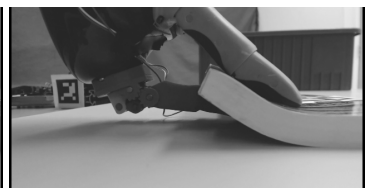

(i)

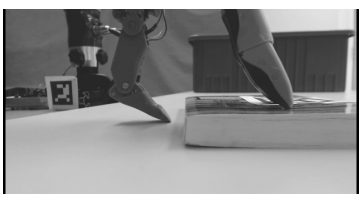

(e)

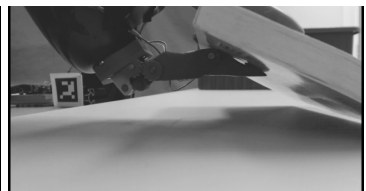

(j)

Figure 20: Grasping of the Book with the edge being detected: (a) Pre-Grasp position (b) Lower (c) Force Trigger (d) Open thumb (e) Edge Detected (f) Adapt (g) Close Thumb (h) Final thumb position achieved (i) Final Grasping (j) Auxiliary task.

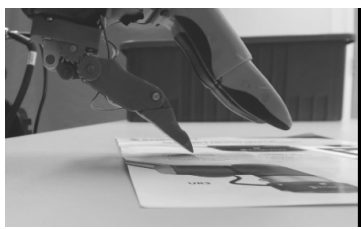

(a)

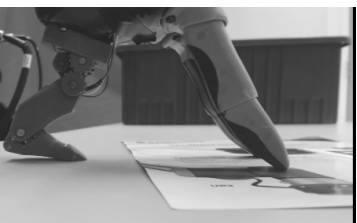

(f)

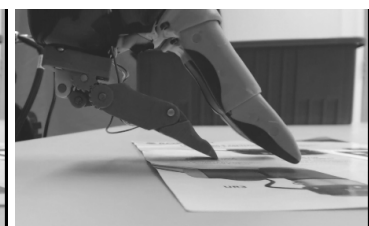

(b)

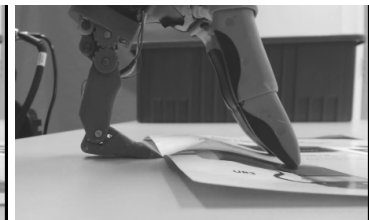

(g)

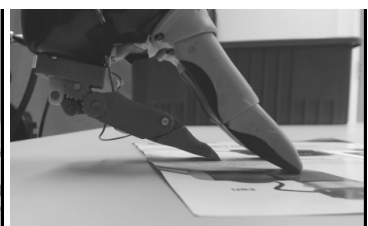

(c)

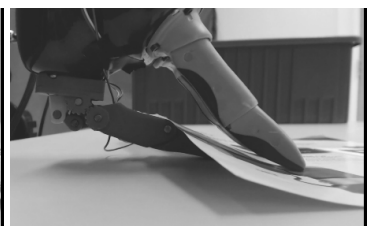

(h)

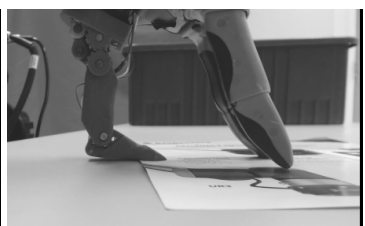

(d)

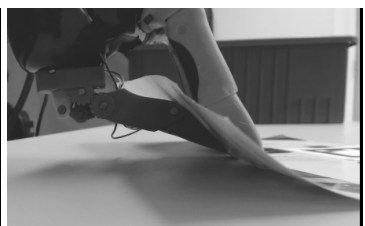

(i)

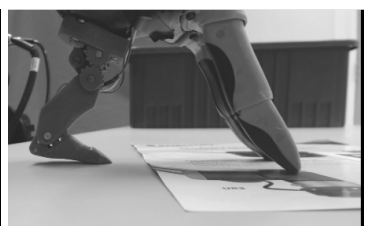

(e)

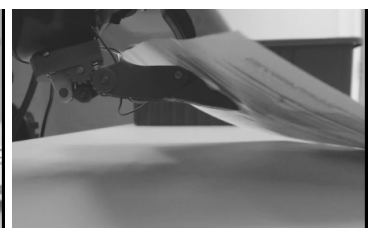

(j)

Figure 21: Grasping of a sheet of paper with the thumb failing to detect the edge: (a) Pre-Grasp position (b) Lower (c) Force Trigger (d) Open thumb (e) Failing to detect the edge (f) Passive joint reaching $\theta_{3}=\theta_{3, \max }$ (g) Close Thumb (h) Final thumb position achieved (i) Final Grasping (j) Auxiliary task.

The accompanying video shows the grasping implemented be graspable and several viewpoints are recorded and shown for the reader to understand the procedure. In order to further demonstrate the repeatability of the method, objects are placed on the table, grasped by the manipulator and moved into a bin in a continuous sequence to show the $100 \%$ success rate of the method with the objects chosen.

\section{Discussion}

The features that make the experiments successful are mostly related to the use of the passive joint to ensure contact with the surface at the tip $P$ of the thumb. This is especially important for the grasping situation addressed in this work because of the nature of the stiffness of the contact with the environment and the position inaccuracies. Uncertainties, in this experiment, mainly arise from three sources. The first is the use of 350 a proportional-derivative (PD) controller to position each joint. This controller is used with relatively low gains in order to prevent damage to the hardware, or sharp initial contacts with the environment. The very stiff environment of this experiment makes it easy to damage the manipulator's fingers. The second source of error is the placement of the camera which is far from both the robot marker and the object marker. This introduces some uncertainty on the position of the tip of the thumb which again is compensated for by the passive joint as an adaptive way to ensure the contact. The third source of error is the imprecise mounting of the manipulator relative to the table surface. The effect of these three sources of error can be observed in the accompanying video, especially in the individual object grasping, by observing that the finger-thumb plane and the object's principal axis do not always coincide.

An interesting observation that was made during the experiments is that the passive thumb's angle being measured, angle $\theta_{3}$ can be used as a way to approach the object at greater speeds. Indeed, when the gripper is lowered towards the object to initiate contact, its speed is limited in order to avoid generating high forces at the fingers. However, using the approach and gripper proposed in this work, the passive thumb makes contact with the object before the fingers. Hence, the measure of the position of the last phalanx of the thumb can be used to reduce the speed to a lower speed upon contact to prepare for the final finger approach. The thumb being significantly more compliant 


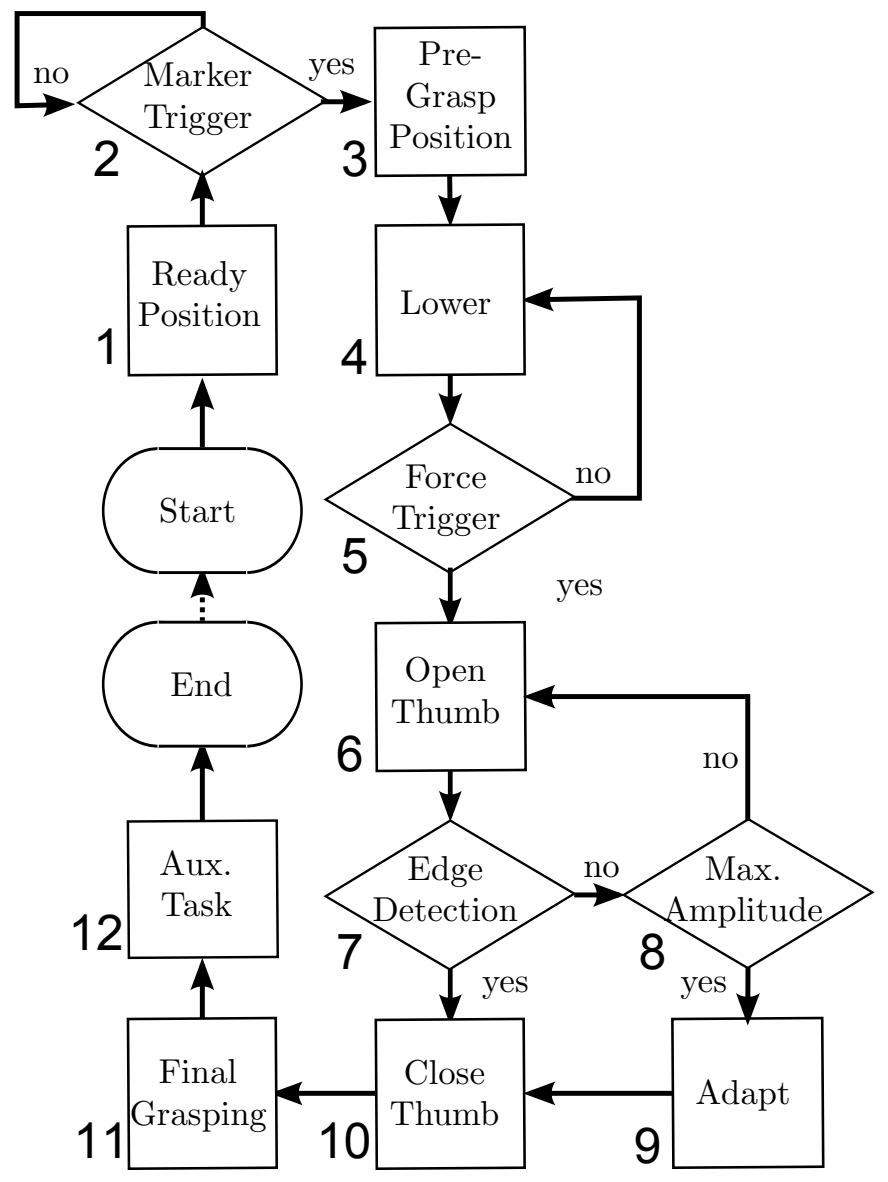

Figure 22: Flow chart of the grasping algorithm.

than the fingers, the reaction time to stop is more favourable. This behaviour is advantageous for two reasons: first by approaching the object faster, delays can be reduced and second by reducing the risk of high force contact more cycles can be expected from the gripper before failure.

Furthermore, as shown in Figure 20, once the edge of the object is detected not only does the extension of the thumb stop but also the adaptation of the orientation of the hand to maximize the thumb's distal phalanx's attack angle. This provides a greater chance of success and also reduces the sequence time as shown in Figure 21 where the object's edge being too thin is not detected and hence the thumb has to reach the maximum value $\theta_{3}=\theta_{3, \text { max }}$ in order to proceed to the insertion phase.

In order to tune the procedure to successfully achieve the

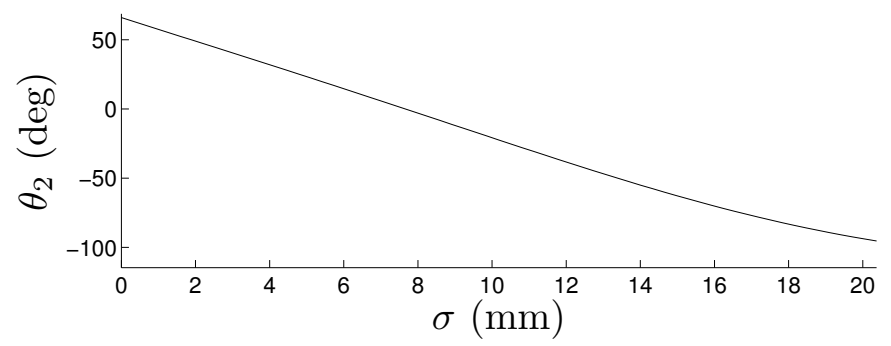

Figure 23: Finger orientation $\left(\theta_{2}\right)$ as a function of the actuator input.

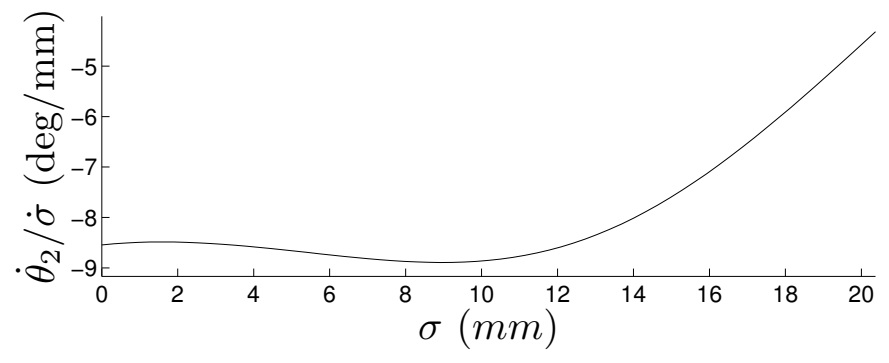

Figure 24: Finger velocity ratio.

grasp, different experimental parameters had to be used for some of the objects, namely the Flexible CD Case and the Rigid CD Case. This can be observed in Table 2 where the finger contact distance $k$ was increased for these objects in order to allow a proper insertion of the thumb. Note that the procedure is successful for all objects with $k=110 \mathrm{~mm}$. As Predicted in Section 2.2.2, increasing the distance $k$ favoured the insertion. It should be pointed out that a larger value of parameter $k$ is used for the booklet only to show in the attached video how far the thumb can reach under a flexible object although the experiment was successful even with smaller values of $k$. It is important to point out that this method largely relies on having the manipulator being able to reach the principal axis of the object and having a favourable workspace to execute the grasp process.

\section{Conclusion}

In this paper, a specific grasping problem, namely the grasping of flat objects resting on a stiff flat surface was addressed. A static model of a rigid object being scooped from a flat surface was first developed. The different parameters involved while artificially generating friction on top of the object and inserting a wedge shaped thumb under it were then studied. The analysis provided insight into the task planning and gripper design. A prototype taking advantage of the results of the analysis was then designed and built and a kinematic analysis of the novel thumb finger was provided. A proof of concept was developed and shown in the accompanying video in order to show the repeatability of the method over a variety of objects. In the accompanying video, each individual object is grasped using the method and a final uninterrupted back to back grasping sequence is provided with all the test objects.

Based on the experimental data, it can be concluded that the grasping procedure is successful in combination with the architecture of the presented prototype, for the set of objects tested. In order to handle positioning uncertainty and consequently compliant robots, it was shown that a passive joint is a very successful option. While useful, the use of a potentiometer for measuring the passive phalanx's position is not essential for the method's success and hence a future version of the gripper may not include it. Future work will also focus on incorporating this procedure to industrial grippers. 


\section{Acknowledgment}

The authors would like to thank the Natural Sciences and Engineering Research Council of Canada (NSERC), Robotiq and Prompt Québec for their financial support.

\section{References}

[1] G. J. Monkman, S. Hesse, R. Steinmann, H. Schunk, Robot grippers, John Wiley \& Sons, 2007.

[2] S. Davis, J. Gray, D. G. Caldwell, An end effector based on the Bernoulli principle for handling sliced fruit and vegetables, Robotics and ComputerIntegrated Manufacturing 24 (2) (2008) 249-257.

[3] V.-D. Nguyen, The synthesis of stable grasps in the plane, in: IEEE International Conference on Robotics and Automation. Proceedings, 1986, Vol. 3, 1986, pp. 884-889.

[4] J.-W. Li, H. Liu, H.-G. Cai, On computing three-finger force-closure grasps of 2-D and 3-D objects, IEEE Transactions on Robotics and Automation 19 (1) (2003) 155-161.

[5] L. Birglen, T. Laliberté, C. M. Gosselin, Underactuated robotic hands, Vol. 40, Springer Tracts on Advanced Robotics, 2007.

[6] M. A. Roa, R. Suárez, Finding locally optimum force-closure grasps, Robotics and Computer-Integrated Manufacturing 25 (3) (2009) 536-544.

[7] F. Reuleaux, E. S. Ferguson, Kinematics of machinery: outlines of a theory of machines, Courier Corporation, 2012.

[8] E. Rimon, J. W. Burdick, Mobility of bodies in contact. I. a 2nd-order mobility index for multiple-finger grasps, IEEE Transactions on Robotics and Automation 14 (5) (1998) 696-708.

[9] M. R. Cutkosky, On grasp choice, grasp models, and the design of hands for manufacturing tasks, IEEE Transactions on Robotics and Automation 5 (3) (1989) 269-279.

[10] A. Bicchi, M. Gabiccini, M. Santello, Modelling natural and artificial hands with synergies, Philosophical Transactions of the Royal Society B 366 (1581) (2011) 3153-3161.

[11] M. G. Catalano, G. Grioli, E. Farnioli, A. Serio, C. Piazza, A. Bicchi, Adaptive synergies for the design and control of the Pisa/IIT softhand, The International Journal of Robotics Research 33 (5) (2014) 768-782.

[12] J. Shintake, S. Rosset, B. Schubert, D. Floreano, H. Shea, Versatile soft grippers with intrinsic electroadhesion based on multifunctional polymer actuators, Advanced Materials 28 (2) (2016) 231-238.

[13] R. Kolluru, K. P. Valavanis, T. M. Hebert, Modeling, analysis, and performance evaluation of a robotic gripper system for limp material handling, IEEE Transactions on Systems, Man, and Cybernetics, Part B (Cybernetics) 28 (3) (1998) 480-486. doi:10.1109/3477.678660.

[14] J. R. Amend, E. Brown, N. Rodenberg, H. M. Jaeger, H. Lipson, A positive pressure universal gripper based on the jamming of granular material, IEEE Transactions on Robotics 28 (2) (2012) 341-350.

[15] L. U. Odhner, R. R. Ma, A. M. Dollar, Precision grasping and manipulation of small objects from flat surfaces using underactuated fingers, in: IEEE International Conference on Robotics and Automation (ICRA), 2012, pp. 2830-2835. doi:10.1109/ICRA.2012.6225211.

[16] M. Ciocarlie, F. M. Hicks, R. Holmberg, J. Hawke, M. Schlicht, J. Gee, S. Stanford, R. Bahadur, The velo gripper: A versatile single-actuator design for enveloping, parallel and fingertip grasps, The International Journal of Robotics Research 33 (5) (2014) 753-767. doi:10.1177/0278364913519148.

[17] K. Kosuge, J. Lee, J. Ichinose, Y. Hirata, A novel grasping mechanism for flat-shaped objects inspired by lateral grasp, in: 2nd IEEE RAS EMBS International Conference on Biomedical Robotics and Biomechatronics, 2008, pp. 282-288. doi:10.1109/BIOROB.2008.4762905.

[18] D. Kappler, L. Y. Chang, N. S. Pollard, T. Asfour, R. Dillmann, Templates for pre-grasp sliding interactions, Robotics and Autonomous Systems 2011.doi:10.1016/j.robot.2011.07.015.

[19] M. Kazemi, J.-S. Valois, J. A. Bagnell, N. Pollard, Human-inspired force compliant grasping primitives, Autonomous Robots 37 (2) (2014) 209225.

[20] M. R. Dogar, S. S. Srinivasa, Push-grasping with dexterous hands: Mechanics and a method, in: International Conference on Intelligent Robots and Systems (IROS), IEEE/RSJ, 2010, pp. 2123-2130.
[21] N. C. Dafle, A. Rodriguez, R. Paolini, B. Tang, S. S. Srinivasa, M. Erdmann, M. T. Mason, I. Lundberg, H. Staab, T. Fuhlbrigge, Extrinsic dexterity: In-hand manipulation with external forces, in: 2014 IEEE International Conference on Robotics and Automation (ICRA), IEEE, 2014, pp. 1578-1585.

[22] A. Sintov, A. Shapiro, Dynamic regrasping by in-hand orienting of grasped objects using non-dexterous robotic grippers, Robotics and Computer-Integrated Manufacturing 50 (2018) 114-131.

[23] Z. Xu, T. Deyle, C. C. Kemp, 1000 trials: An empirically validated end effector that robustly grasps objects from the floor, in: Robotics and Automation, 2009. ICRA'09. IEEE International Conference on, IEEE, 2009, pp. 2160-2167.

[24] C. Eppner, R. Deimel, J. Álvarez-Ruiz, M. Maertens, O. Brock, Exploitation of environmental constraints in human and robotic grasping, The International Journal of Robotics Research, vol. 34, no. 7, pp. 1021-1038, 2015 .

[25] A. I. H. Committee, ASM handbook: Friction, lubrication, and wear technology, Vol. 18, ASM International, 1992.

[26] V. Babin, C. Gosselin, J.-F. Allan, A dual-motor robot joint mechanism with epicyclic gear train, in: 2014 IEEE/RSJ International Conference on Intelligent Robots and Systems, 2014, pp. 472-477.

[27] S. Garrido-Jurado, R. Muñoz-Salinas, F. Madrid-Cuevas, R. MedinaCarnicer, Generation of fiducial marker dictionaries using mixed integer linear programming, Pattern Recognition 51 (2016) 481-491.

[28] S. Garrido-Jurado, R. Muñoz-Salinas, F. J. Madrid-Cuevas, M. J. MarínJiménez, Automatic generation and detection of highly reliable fiducial markers under occlusion, Pattern Recognition 47 (6) (2014) 2280-2292. 